\title{
IMPURITY
} CENTERS

\section{Paramagnetic Defects in Manganese-Doped Lead Tungstate}

\author{
G. R. Asatryan ${ }^{a, *}$, M. Nikl ${ }^{b}$, V. A. Vazhenin ${ }^{c, * *}$, and A. P. Potapov ${ }^{c}$ \\ ${ }^{a}$ Ioffe Physical-Technical Institute, Russian Academy of Sciences, Politekhnicheskaya ul. 26, St. Petersburg, 194021 Russia \\ *e-mail: hike.asatryan@mail.ioffe.ru \\ ${ }^{b}$ Institute of Physics, Czech Academy of Science, Na Slovance 1999/2, Prague 8, 18221 Czech Republic \\ ${ }^{c}$ Institute of Natural Science, Ural Federal University named after the First President of Russia B.N. Yeltsin, \\ ul. Kuibysheva 48, Yekaterinburg, 620083 Russia \\ **e-mail: vladimir.vazhenin@usu.ru \\ Received May 10, 2012
}

\begin{abstract}
In manganese-doped $\mathrm{PbWO}_{4}$ crystals, low-intensity signals of triclinic clusters $\mathrm{Mn}^{4+}-V_{\mathrm{O}}$ and $\mathrm{Fe}^{3+}-V_{\mathrm{Pb}}$ have been revealed in addition to signals of $\mathrm{Mn}^{2+}$ tetragonal centers. The $\mathrm{Mn}^{4+}-V_{\mathrm{O}}$ cluster is formed by a $\mathrm{Mn}^{4+}$ ion in the $\mathrm{W}^{6+}$ position, which is associated with a vacancy of the nearest neighbor $\mathrm{O}^{2-}$ ion, and the $\mathrm{Fe}^{3+}-V_{\mathrm{Pb}}$ cluster consists of a $\mathrm{Fe}^{3+}$ ion substituting for $\mathrm{Pb}^{2+}$ with a local compensation of by a lead vacancy. It has been shown that, in $\mathrm{PbWO}_{4}: \mathrm{Mn}$, there is also a small amount of $\mathrm{Mn}^{4+}$ tetragonal centers located in the $\mathrm{Pb}^{2+}$ position with a nonlocal compensation of an excess charge.
\end{abstract}

DOI: $10.1134 / \mathrm{S} 106378341301006 \mathrm{X}$

\section{INTRODUCTION}

Single crystals of lead tungstate $\mathrm{PbWO}_{4}$ (PWO) with a high density $\left(8.27 \mathrm{~g} / \mathrm{cm}^{3}\right)$ are known as fast scintillator materials, which have successfully been used in modern high-energy physics experiments (see [1-4] and references therein). By doping with trivalent ions, these crystals were optimized for ALICE and PANDA calorimetric detectors [5, 6]. About ten years ago, research had been focused on an increase in the quantum yield $[7,8]$ and on the development of various doping schemes and annealing procedures [9-12]. Progress in increasing the quantum yield was achieved by means of the double doping of PWO crystals with Mo and La ions, as well as with $\mathrm{Y}$ and $\mathrm{Nb}$ ions $[7,8,13,14]$. This doping increases the quantum yield by a factor of 2-4 without a significant deterioration of other scintillation characteristics. More recently, considerable interest has arisen in the socalled dual readout approach for future generations of electromagnetic calorimeters [15], in which scintillations and Cherenkov radiation are controlled simultaneously. In this aspect, $\mathrm{PbWO}_{4}$ crystals were investigated in [16, 17].

Another application of PWO is associated with the use of scintillator materials at very low temperatures (mK) $[18,19]$. In $\mathrm{PbWO}_{4}$, intrinsic luminescence and scintillations have an excitonic nature, and the charge transfer band in complex oxyanions $\left(\mathrm{WO}_{4}\right)^{2-}$ leads to the emission in the blue spectral region [20]. Selftrapped excitons are destroyed even at a temperature of $\sim 150 \mathrm{~K}$ [21]. Therefore, at room temperature, any levels of shallow traps in the PWO lattice are involved in the capture processes and become very important.
They change the characteristics of the migration of free charge carriers by means of the recapture in which any uncontrollable impurities or defects can be of considerable importance. Laguta et al. [22-25] described and explained in detail the electron cascade in PWO. Finally, hole traps of several configurations in doubly doped PWO : Mo,La crystals have recently been discovered in [26]. The successful use of $\mathrm{PbWO}_{4}$ crystals in the aforementioned applications requires a deeper understanding of the luminescence mechanisms and, therefore, knowledge of the structure of defects and uncontrollable impurities generating features of the charge carrier capture in the $\mathrm{PbWO}_{4}$ structure.

Electron paramagnetic resonance (EPR) is one of the most direct methods used for studying the structure of impurity centers in quantum electronics [27]. The EPR method makes it possible to determine the spin, charge state of an impurity, local symmetry of a center, structure of the nearest environment, and specific features of the interaction with the crystal lattice. Information on papers containing results of magnetic resonance investigations of impurity centers, such as $\mathrm{Nd}^{3+}, \mathrm{Ce}^{3+}, \mathrm{Gd}^{3+}, \mathrm{Mn}^{2+}, \mathrm{Fe}^{3+}, \mathrm{Cr}^{3+}$, and other paramagnetic defects in $\mathrm{PbWO}_{4}$, can be found in $[25$, 28-30].

A detailed study using the method of paramagnetic resonance of a tetragonal center of the $\mathrm{Mn}^{2+}$ ion (electron spin $S=5 / 2$, nuclear spin $I=5 / 2$ ) located in the position of the $\mathrm{Pb}^{2+}$ ion in lead tungstate at temperatures in the range from 1.5 to $290.0 \mathrm{~K}$ was carried out in $[31,32]$. 

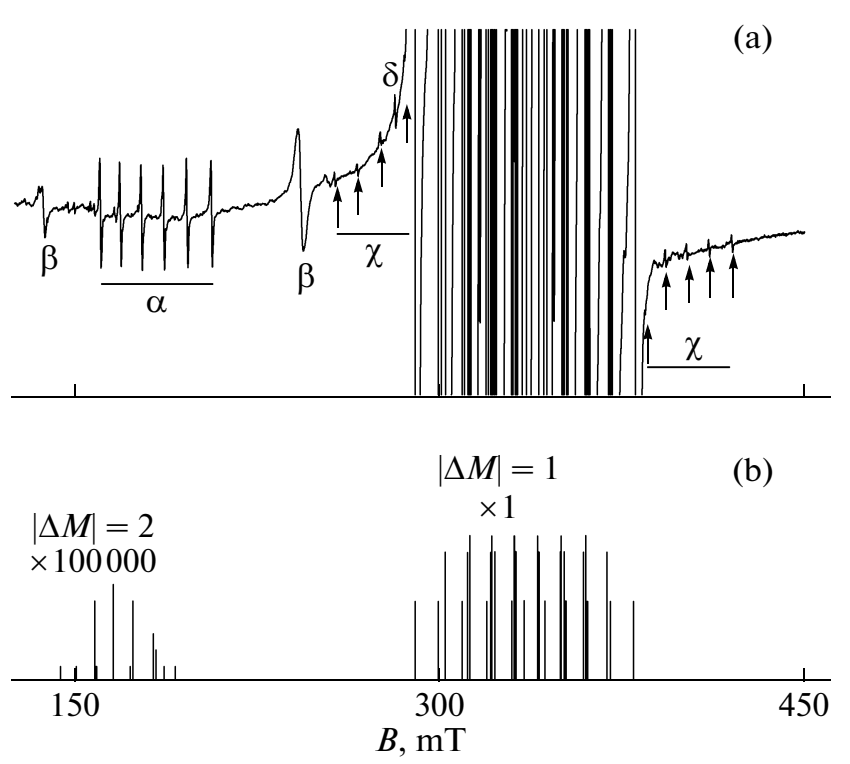

Fig. 1. (a) EPR spectrum (derivative of the absorption signals) of $\mathrm{PbWO}_{4}:$ Mn for the orientation $\mathbf{B} \| \mathbf{S}_{4}$ at $100 \mathrm{~K}$. Intense signals in the range of magnetic fields 284-386 mT belong to transitions $(|\Delta M|=1)$ of the $\mathrm{Mn}^{2+}$ tetragonal center located in the $\mathrm{Pb}^{2+}$ position $[31,32]$. (b) Calculated spectrum of transitions of the $\mathrm{Mn}^{2+}$ tetragonal center.

This paper is devoted to the magnetic resonance investigation of other paramagnetic defects realized in $\mathrm{PbWO}_{4}: \mathrm{Mn}$.

\section{SAMPLE PREPARATION AND EXPERIMENTAL TECHNIQUE}

The measurements were carried out on electron paramagnetic resonance spectrometers Bruker EMX Plus and JEOL operating in the X-band at temperatures in the range from 4 to $300 \mathrm{~K}$ in magnetic fields up to $1.45 \mathrm{~T}$ with the use of lead tungstate crystals investigated in [31, 32], according to which the crystals were doped with tin and bismuth in addition to manganese.

It is known that the tungsten ion in the $\mathrm{PbWO}_{4}$ crystal is located in a slightly distorted oxygen tetrahedron and that the environment of the lead ion consists of two nested oxygen tetrahedra, one of which is elongated and the other one is compressed along the tetragonal axis. The space group of the crystal at temperatures of $300 \mathrm{~K}$ and $1.4 \mathrm{~K}$ is $I 4_{1} / a\left(C_{4 h}^{6}\right)$, and the local symmetry of the $\mathrm{Pb}^{2+}$ and $\mathrm{W}^{6+}$ positions is $S_{4}[33,34]$. In this respect, the assertion made by the authors of $[23,30]$ that the position of the ion tungsten has the symmetry group $D_{2 d}$ is obviously erroneous. Taking into account the results reported in $[31,32]$, the statement made in [30] that the parameters of the spin Hamiltonian of $\mathrm{Mn}^{2+}$ centers do not depend on the temperature seems to be strange.

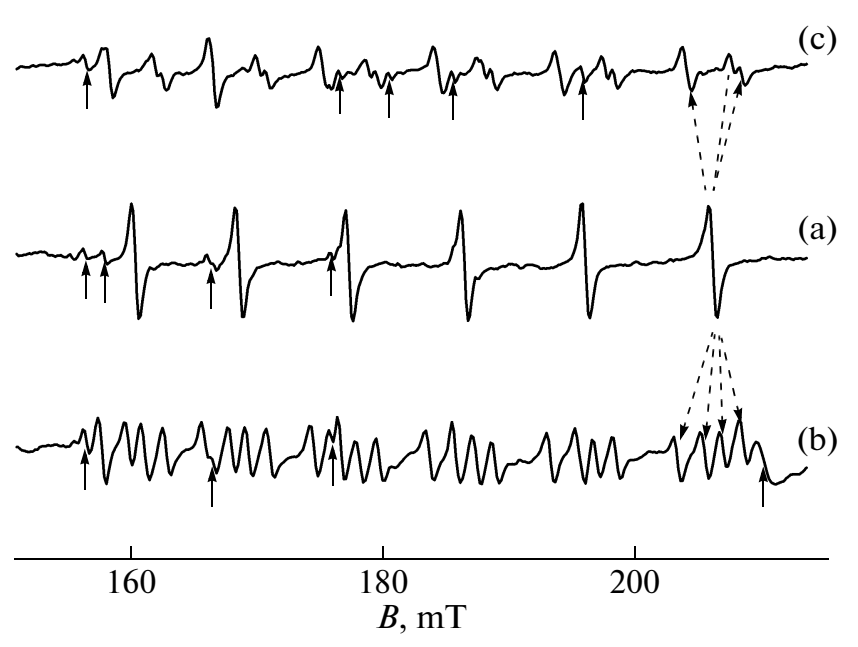

Fig. 2. Splitting of the spectrum of the $\alpha$ center upon deviation from the orientation $\mathbf{B} \| \mathbf{S}_{4}$ in different planes at $T=$ $100 \mathrm{~K}$ : (a) spectrum at $\mathbf{B} \| \mathbf{S}_{4}$; (b) spectrum at $\theta=6^{\circ}, \varphi=$ $40^{\circ}$; and (c) spectrum at $\theta=5.5^{\circ}, \varphi=0^{\circ}$. Dashed arrows show the splitting for one of the hyperfine components as an example. Solid arrows indicate signals not related to the $\alpha$ center. The axes of the spherical coordinate system coincide with the crystallographic axes.

\section{RESULTS AND DISCUSSION}

3.1. In addition to the intense spectra of $\mathrm{Mn}^{2+}$ tetragonal centers $[31,32]$ in the studied samples of $\mathrm{PbWO}_{4}$, we revealed very weak spectra of at least three centers $(\alpha, \beta, \chi)$ shown in a fragment of the EPR spectrum (Fig. 1a) measured for the magnetic field orientation $\mathbf{B}\|c\| \mathbf{S}_{4}$ (B is the magnetic induction) at a temperature of $100 \mathrm{~K}$ and a frequency of $9423 \mathrm{MHz}$.

The sextet of the $\alpha$ center (Fig. 1a) is also observed at room temperature. A characteristic feature of this spectrum is the splitting of all signals upon deviation from the orientation $\mathbf{B} \| \mathbf{S}_{4}$ into four components (Fig. 2). It is in this range of magnetic fields that there should exist four forbidden $(\Delta M= \pm 2$, where $M$ is the projection of the electron spin) transitions of the $\mathrm{Mn}^{2+}$ tetragonal center, which naturally have a hyperfine structure.

In order to answer the question as to whether the signals of the sextet are due to the aforementioned forbidden transitions, we calculated their spectra with the parameters of the spin Hamiltonian [32]. The calculations demonstrated that the forbidden transitions do not lead to the formation of a regular sextet (Fig. 1b), and their signals do not split into four components of comparable intensity upon deviation from the orientation $\mathbf{B} \| \mathbf{S}_{4}$. These transitions are responsible for still weaker signals observed within and below the sextet of the $\alpha$ center in Figs. 1 and 2.

The fact of the merger of four EPR spectra for the tetragonal crystal $I 4_{1} / a\left(C_{4 h}^{6}\right)$ in the orientation of the magnetic field $\mathbf{B} \| \mathbf{S}_{4}$ clearly indicates that these cen- 
ters has the $C_{1}$ symmetry. This type of centers in the crystal can arise as a result of the compensation for an excess charge of the paramagnetic ion by a charged defect located in a closely spaced position. The multiplicity of such centers in crystals with the scheelite structure is 8; however, because four of them are related to the four others by the inversion operation, the magnetic multiplicity is 4 .

Other transitions of the $\alpha$ center for the orientation B $\| \mathbf{S}_{4}$ over the entire range of magnetic fields were not found. Therefore, in order to determine the charge state of the manganese ion, which is responsible for the sextet of the $\alpha$ center, we considered its hyperfine splitting. The measured average splitting $(253 \mathrm{MHz})$ counts in favor of the fact that it belongs to the $\mathrm{Mn}^{4+}$ center $(S=3 / 2)$; a similar value for the $\mathrm{Mn}^{2+}$ center in $\mathrm{PbWO}_{4}$ is $267 \mathrm{MHz}$ [32]. In this case, there are two variants of the model of the $\alpha$ center: (1) the $\mathrm{Mn}^{4+}$ ion substitutes for the $\mathrm{Pb}^{2+}$ ion, and the charge is compensated by means of the formation of a vacancy of the lead ion $V_{\mathrm{Pb}}\left(R=4.055 \AA, \theta=42^{\circ}\right.$, and $\varphi=0^{\circ}$ are the coordinates of the $\mathrm{Pb}^{2+}$ ion from the nearest lead tetrahedron in the $a b c$ crystallographic system centered on the impurity ion); and (2) the $\mathrm{Mn}^{4+}$ ion located in the $\mathrm{W}^{6+}$ position is associated with the vacancy of the oxygen ion $V_{\mathrm{O}}\left(R=1.775 \AA, \theta=56.15^{\circ}\right.$, and $\varphi=31^{\circ}$ are the coordinates of the $\mathrm{O}^{2-}$ ion involved in the nearest oxygen tetrahedron). In both cases, there arise four triclinic centers, which transform into each other upon rotation around $\mathbf{S}_{4}$ through angles $p \cdot 90^{\circ}$ ( $p$ is an integer).

The vacancies of lead and oxygen ions, which are located in the aforementioned positions, induce a strong electric field at the paramagnetic ion. Therefore, these centers can be approximately considered to be axial with the principal magnetic axis directed along the bond of the dimer cluster. Then, upon rotation of the magnetic field in the plane containing $\mathbf{S}_{4}$ and the dimer axis, we should expect the splitting of all signals of the sextet into three components, and the central component should be double. As can be seen from Fig. 2, this character of the splitting of the components in the spectrum for the $\alpha$ center takes place upon rotation of the magnetic field in the plane with $\varphi=40^{\circ}$ rather than in the plane with $\varphi=0^{\circ}$, where the splitting occurs practically into two double components. The observed splitting of the central component in Fig. 2 at $\varphi=40^{\circ}$ can be due to both the approximate assumption of the axiality of the centers and the mismatch between the studied plane and the plane containing $\mathbf{S}_{4}$ and the dimer axis.

Although manganese quasi-sextets are observed in some arbitrary orientations of the magnetic field above the group of signals due to the $\mathrm{Mn}^{2+}$ tetragonal center, we could not measure extended angular dependences of other transitions of the $\alpha$ center, which allow their identification. The measurements are difficult to per- form because of both the low intensity of the signals and the appearance of forbidden hyperfine transitions resulting in a distortion of the canonical manganese sextets. Furthermore, in arbitrary orientations of the magnetic field, we observed groups of signals with close intensities at intervals approximately two times shorter than those in the spectrum of individual manganese ions. Most probably, these signals belong to pairs of closely spaced tetragonal centers $\mathrm{Mn}^{2+}$.

Thus, at present, we can only argue that the $\alpha$ center, most likely, is formed by the $\mathrm{Mn}^{4+}$ ion, which substitutes for the $\mathrm{W}^{6+}$ ion and has an oxygen vacancy in the nearest environment.

3.2. In contrast to the $\alpha$ center, the spectrum of the $\chi$ center in $\mathrm{PbWO}_{4}$ consists of two incomplete sextets (indicated by arrows in Fig. 1a), whose components exhibit extrema of the resonance positions for the orientation $\mathbf{B} \| \mathbf{S}_{4}$ and, therefore, belong to the tetragonal center. The EPR signals in the spectrum of the $\chi$ center can be observed outside the spectrum of the intense $\mathrm{Mn}^{2+}$ tetragonal center in only a small $\left( \pm 9^{\circ}\right)$ neighborhood of $\mathbf{B} \| \mathbf{S}_{4}$. The value of the hyperfine splitting of these sextets indicates that they belong to the $\mathrm{Mn}^{4+}$ center, which is described by the spin Hamiltonian $(S=3 / 2, I=5 / 2)$ in the following form [27]:

$$
H_{\mathrm{sp}}=\mathbf{g} \beta(\mathbf{B S})+\frac{1}{3} b_{20} O_{20}+\mathbf{A}(\mathbf{S I}),
$$

where $\mathbf{g}$ is the $g$-tensor; $\beta$ is the Bohr magneton; $\mathbf{S}$ and $I$ are the electron and nuclear spin operators, respectively; $b_{n m}$ are the fine structure parameters; $O_{n m}$ are the Stevens spin operators; and $\mathbf{A}$ is the hyperfine coupling tensor.

The optimization of the parameters taking into account the orientation behavior of the components of the sextets assigned to the electronic transitions $\pm 1 / 2 \longleftrightarrow \pm 3 / 2$ led to the values $g=1.983(2), b_{20}=$ $-815(5) \mathrm{MHz}$, and $A=-253(3) \mathrm{MHz}$ (the rootmean-square deviation is $10 \mathrm{MHz}$ ) for the orientation $\mathbf{z} \| \mathbf{S}_{4}$ at $T=100 \mathrm{~K}$. It can be seen that the parameters $g$ and $A$ have characteristic values for the $\mathrm{Mn}^{4+}$ centers [27]. With an increase in the temperature, the absolute value of $b_{20}$ increases $\left(\left|d b_{20} / d T\right| \approx 2 \mathrm{MHz} / \mathrm{K}\right)$.

The presence of the $\delta$ signal at the minimum resonance position in the spectrum (Fig. 1a) for the orientation $\mathbf{B} \| \mathbf{S}_{4}$ initiates the following assumption: the $\delta$ signal is assigned to the low-field hyperfine component of the transition $\pm 1 / 2 \longleftrightarrow \pm 3 / 2$ of the center with spin $S=5 / 2$, and the sextets of the $\chi$ center are attributed to the transitions $\pm 3 / 2 \longleftrightarrow \pm 5 / 2$. The description of the spectrum of the $\chi$ center under this assumption by the spin Hamiltonian, which, in addition to Hamiltonian (1), includes the fourth-rank terms,

$$
\frac{1}{60} b_{40} O_{40}+\frac{1}{60} b_{44} O_{44}
$$


(a)

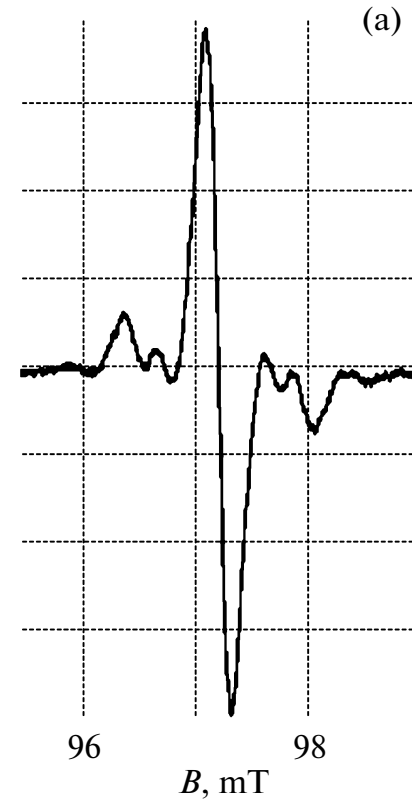

(b)

Fig. 3. Superhyperfine structure of the low-field component in the spectrum of the $\beta$ center for the parameters $T=$ $28 \mathrm{~K}, \theta \approx 75^{\circ}$, and $\varphi=0^{\circ}$ : (a) experiment and (b) simulation.

gives the following parameters: $g=1.981, b_{20}=$ $-400 \mathrm{MHz}, b_{40}=-8 \mathrm{MHz},\left|b_{44}\right| \geq 5500 \mathrm{MHz}$, and $A=$ $-255 \mathrm{MHz}$. And, in this case, also, the parameters $g$ and $A$ have characteristic values for the $\mathrm{Mn}^{4+}$ centers.

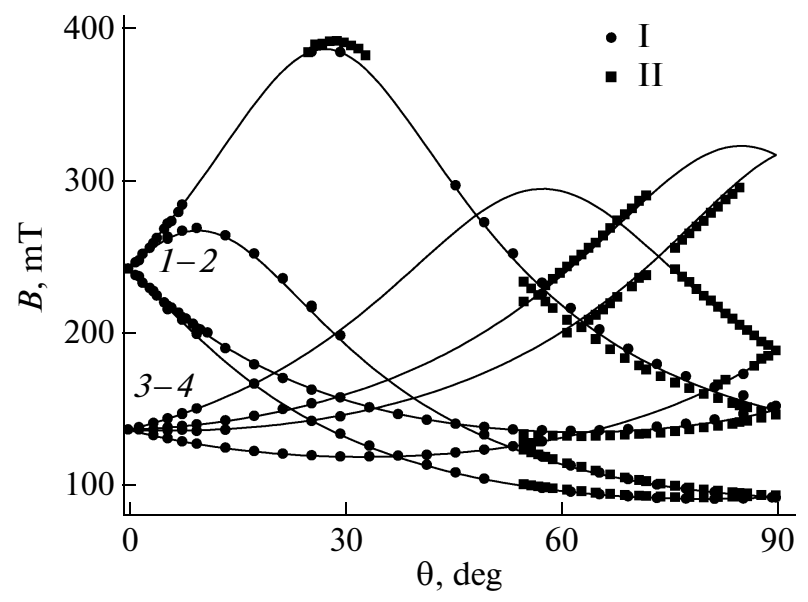

Fig. 4. Orientation behavior of the positions of the EPR signals from the $\beta$ center in the $c a$ plane. Symbols I and II represent the experimental results obtained in different cycles of measurements. Curves correspond to the results of the calculations with the parameters given in the table (variant 1). (1-2), (3-4) Energy levels between which there occur transitions of four triclinic centers equivalent to each other for the orientation $\mathbf{B} \| \mathbf{S}_{4}$. The numbering of levels is given in order of increasing energy.
Consequently, the $\chi$ center should be considered as a tetragonal center with spin 3/2; i.e., it is the $\mathrm{Mn}^{4+}$ ion.

The zero-field splitting parameter of the $\chi$ center (with $S=3 / 2$ ) is 5.7 times greater than that of the ground-state tetragonal center $\mathrm{Mn}^{2+}[31,32]$. The closeness of this value to the ratio of the parameters $b_{20}\left(\mathrm{Mn}^{4+}\right) / b_{20}\left(\mathrm{Mn}^{2+}\right) \approx 9$ in $\mathrm{Al}_{2} \mathrm{O}_{3}$ [27] and $b_{20}\left(\mathrm{Mn}^{4+}\right) / b_{20}\left(\mathrm{Mn}^{2+}\right) \approx 5.3$ in $\mathrm{LaGaO}_{3}[35,36]$ for the manganese centers located in the same position of the crystal is a strong argument in favor of the location of the discussed center $\mathrm{Mn}^{4+}$ in the $\mathrm{Pb}^{2+}$ position with a nonlocal compensation of an excess positive charge.

3.3. EPR signals of the $\beta$ type (Fig. 1a), which at liquid-helium temperature exhibit approximately the same superhyperfine structure (Fig. 3), should be assigned to transition of the same paramagnetic center. A characteristic feature of this spectrum is the disappearance of the signals at $T>130 \mathrm{~K}$ and their splitting upon deviation from the orientation $\mathbf{B} \| \mathbf{S}_{4}$ into four components. Most likely, the discussed paramagnetic center with the $C_{1}$ symmetry, as well as the $\alpha$ center, is associated with a local compensation of the excess charge of the impurity. The angular dependences of the resonance positions of transitions of the $\beta$ center in two crystallographic planes ( $c a$ and $a b$ ) are shown in Figs. 4 and 5. In both figures, intense signals of the $\mathrm{Mn}^{2+}$ ions cover the magnetic field range from 300 to $380 \mathrm{mT}$. Each signal of the azimuthal dependence (Fig. 5) is a superposition of two transitions, and their splitting at $\varphi>120^{\circ}$ is caused by a small deviation of the magnetic field from the $a b$ plane. It should be noted that these dependences are observed in magnetic fields close to the position of the only signal from the $\mathrm{Fe}^{3+}$ ion detected by the authors of [37] in scheelite $\mathrm{CaWO}_{4}$ with the factor $g_{\text {eff }} \approx 4.3$ for the orientation $\mathbf{B} \| \mathbf{S}_{4}$. In addi-

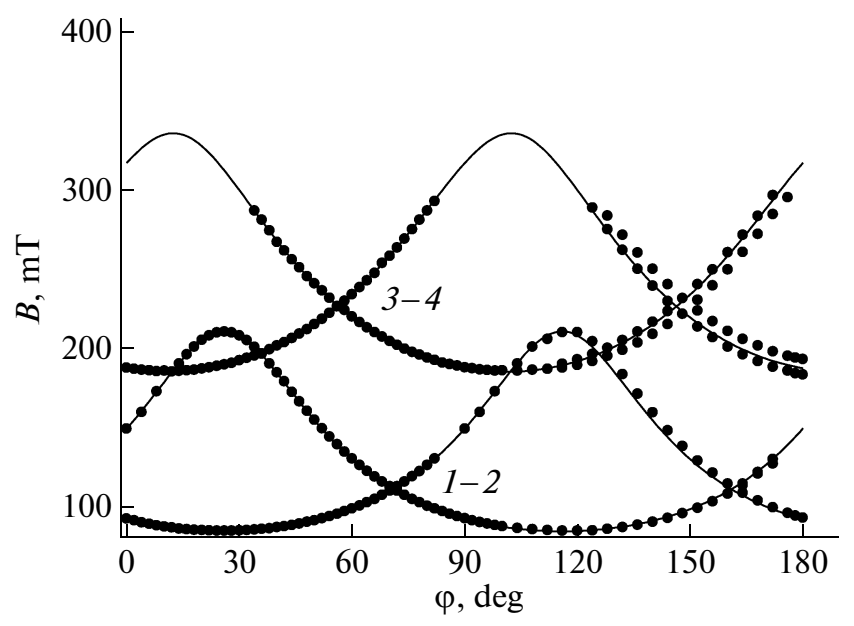

Fig. 5. Angular dependences of the resonance positions of the transitions of the $\beta$ center in the $a b$ plane. Points are the experimental data, and curves represent the results of the calculations. $(1-2),(3-4)$ The same as in Fig. 4. 
Parameters of the triclinic spin Hamiltonian $(S=5 / 2)$ of the $\beta$ center $\left(\mathbf{z}\left\|\mathbf{S}_{4}, \mathbf{x}\right\| \mathbf{a}, \mathbf{y} \| \mathbf{b}, T=100 \mathrm{~K}\right)$ : the root-meansquare deviation $F, b_{n m}$ and $c_{n m}$ (in $\mathrm{MHz}$ ), and $n$ is the number of experimental resonance positions taken into account in the procedure of computer optimization of the parameters

\begin{tabular}{c|c|c|c}
\hline Parameter & Variant 1 & Variant 2 & $\begin{array}{c}\theta=32.8^{\circ}, \\
\varphi=-9.2^{\circ}\end{array}$ \\
\hline$b_{20}$ & 21950 & 31830 & 37070 \\
$b_{21}$ & 93560 & 135540 & -0.05 \\
$b_{22}$ & 20400 & 29500 & 8650 \\
$c_{21}$ & -7180 & -10890 & -0.19 \\
$c_{22}$ & -13510 & -19560 & -7430 \\
$n$ & 309 & 309 & \\
$F$ & 54 & 65 & \\
\hline
\end{tabular}

tion, the character of the angular dependences presented in Figs. 4 and 5 is qualitatively consistent with the behavior of components of the signal from the $\mathrm{Fe}^{3+}$ ion in $\mathrm{CaWO}_{4}$, which is slightly $(\sim 0.7 \mathrm{mT})$ split in an arbitrary orientation of the magnetic field into four components.

The nature of the paramagnetic center of iron in calcium tungstate and the features of the EPR spectrum of this center were discussed, in particular, in [37-41]. The authors of [38] showed that the observed nearly isotropic EPR signal can be explained by a transition in the middle (numbers of energy levels 3-4) doublet of the $\mathrm{Fe}^{3+}$ center under the condition of the approximate equality of the spin Hamiltonian parameters $b_{20} \equiv D$ and $b_{22} \equiv 3 E$ when $b_{20}>g \beta B$. In this case, the transition in the lower $(1-2)$ doublet $\left(b_{20}>0\right)$ is forbidden, whereas the transition in the upper (5-6) doublet is predicted in very large fields. In order to improve the quality of the description of the spectrum, the authors of $[38,39]$ took into account the fourthrank zero-field splitting parameters, and the authors [40] considered terms of the Zeeman interaction of the $S^{3} B$ and $S^{5} B$ types. In [39], among other variants, the authors discussed the possibility of substituting the $\mathrm{Fe}^{3+}$ ion for the calcium position with the compensation of the excess charge by the $\mathrm{Ca}^{2+}$ vacancy. The understanding of the nature of the nearly isotropic spectrum of the $\mathrm{Fe}^{3+}$ ion is important due to the existence of an EPR signal with $g_{\text {eff }} \approx 4.3$ in disordered (including biological) media [41]. It should be noted that, in $\mathrm{PbWO}_{4}: \mathrm{Fe}^{3+}$, the authors of [42] revealed a center with $S=5 / 2$ and described its by the rhombic spin Hamiltonian. It is unclear why such a low-symmetry center is not manifolded by the symmetry operation $\left(S_{4}\right)$ of the crystal.

The spectrum of the $\beta$ center observed in $\mathrm{PbWO}_{4}$ was described by the triclinic spin Hamiltonian $(S=$ $5 / 2$ ), which, in addition to Hamiltonian (1), contains the off-diagonal operators of the fine structure with the assumption of the isotropic factor $g=2$. The results obtained are presented in the table (variant 1) and in Figs. 4 and 5. As can be seen, the angular dependences of the resonance positions in two planes with a good accuracy are described by only the second-rank parameters; in this case, we have $b_{20} / g \beta B \approx$ $2.3(g \beta B=v$, where $v$ is the used microwave frequency). The addition of fourth-rank terms of type (2) to the Hamiltonian insignificantly decreases the rootmean-square deviation.

The lack of information about the value of the zero-field splittings (i.e., about the position of interdoublet transitions) in the experimental data leads to an ambiguity of the parameters of the $\beta$ center. For example, variant 2 of the zero-field splitting parameters (see table), which was obtained by the computer optimization and starts with the values $b_{2 m}$ increased by a factor of 1.5 as compared to the parameters of variant 1 , with a comparable accuracy describes the observed spectrum. This procedure makes it possible to obtain a number of sets of parameters that satisfy the experiment. The description of the spectrum is significantly (by more than 20\%) deteriorated for sets of parameters with $3.5<b_{20} / g \beta B<1.8$. The approximate equality of the parameters $b_{20}$ and $b_{22}$ in all sets ensures the existence of a nearly isotropic transition in the middle (3-4) doublet [38]. The parameters $b_{21}, c_{21}$, and $c_{22}$ determine a character of the orientation behavior of its components and increase the probability of the transition in the lower (1-2) doublet.

Because the spectrum of the $\beta$ center disappears with an increase in the temperature and the description of its angular dependences is possible only taking into account the second-rank parameters, and because of the lack of reliable evidence that the spectrum of the $\mathrm{Fe}^{3+}$ ion (or the center with $S=5 / 2$ ) is detected, an attempt was made to explain it in terms of the spin Hamiltonian with $S=3 / 2$. The best rootmean-square deviation obtained under this assumption with variations in all the tensor components $b_{2 m}$, as well as in three components of the $g$-tensor, was found to be larger than $350 \mathrm{MHz}$. Therefore, this variant cannot be considered to be possible.

When the $z$ axis of the used coordinate system is rotated in the direction with the coordinates $\theta=32.8^{\circ}$ and $\varphi=-9.2^{\circ}$, the second-rank zero-field splitting tensor (variant 1 ) of the $\beta$ center becomes almost diagonal (see table), and the parameter $b_{20}$ has a maximum. The direction thus determined (the magnetic axis of the center), most likely, is associated with a charged defect located near it. Possible models of the localization and compensation of $\mathrm{Fe}^{3+}$ centers in $\mathrm{PbWO}_{4}$ coincide with the model proposed in item 3.1 for $\mathrm{Mn}^{4+}$ dimer clusters. As can be seen, the orientation of the magnetic axis of the center is in better agreement with the coordinates of the $\mathrm{Pb}^{2+}$ vacancy $\left(\theta=42^{\circ}, \varphi=0^{\circ}\right)$ in the nearest environment of the 
lead ion with respect to the $\mathrm{Pb}^{2+}$ position as compared to the direction from the $\mathrm{W}^{6+}$ position to the oxygen vacancy $\left(\theta=56.15^{\circ}, \varphi=31^{\circ}\right)$. Therefore, the $\mathrm{Fe}^{3+}$ ion in $\mathrm{PbWO}_{4}$ substitutes for the $\mathrm{Pb}^{2+}$ ion with a local compensation of the charge by the nearest neighbor lead vacancy.

In this variant of the localization and charge compensation of $\mathrm{Fe}^{3+}$ ions in $\mathrm{PbWO}_{4}$, we should expect a noticeable distortion of the environment, including the environment of lead, and the interaction with its nuclei, most likely, is responsible for the structure shown in Fig. 3a. As a result, the electron-nucleus interactions with the three remaining ${ }^{20} \mathrm{~Pb}$ will be different. This is also evidenced by good agreement between the experimental and simulated structures (Fig. 3b) under the assumption of the interaction with three nonequivalent nuclei of $\mathrm{Pb}^{2+}$ with the interaction parameters $A_{1}=1.6 \mathrm{mT}, A_{2}=1.1 \mathrm{mT}$, and $A_{3}=$ $0.4 \mathrm{mT}$.

\section{CONCLUSIONS}

Thus, the doping of lead tungstate with manganese results predominantly to the substitution of $\mathrm{Mn}^{2+}$ ions for lead positions. A smaller (by more than three orders of magnitude) part of the impurity in the crystal exists in the form of quadruply charged manganese. The $\mathrm{Mn}^{4+}$ ions occupy positions of both the $\mathrm{Pb}^{2+}$ ions with a nonlocal charge compensation and the $\mathrm{W}^{6+}$ ions, thus creating a vacancy of the nearest neighbor oxygen. The weak EPR signals observed for the orientation B $\| \mathbf{S}_{4}$ in magnetic fields of 137 and $243 \mathrm{mT}$ should be attributed to transitions of the $\mathrm{Fe}^{3+}$ ion contained in the samples as an uncontrollable impurity, located in the $\mathrm{Pb}^{2+}$ position, and associated with a lead vacancy.

\section{ACKNOWLEDGMENTS}

We would like to thank P.G. Baranov, M.Yu. Artyomov, and A.D. Gorlov for their interest expressed in this work.

This study was supported by the Ministry of Education and Science of the Russian Federation (state contract nos. 14.740.11.0048 and 16.513.12.3007) and the Russian Academy of Sciences within the framework of the programs "Spin Phenomena in Solid-State Nanostructures and Spintronics" and "Fundamental Principles of Technologies of Nanostructures and Nanomaterials."

\section{REFERENCES}

1. V. G. Baryshevski, M. Korzhik, V. I. Moroz, V. B. Pavlenko, A. F. Lobko, A. A. Fedorov, V. A. Kachanov, V. L. Solovjanov, B. I. Zadneprovsky, V. A. Nefyodov, P. V. Nefyodov, B. A. Dorogovin, and L. L. Nagornaja,
Nucl. Instrum. Methods Phys. Res., Sect. A 322, 231 (1992).

2. M. Kobayashi, M. Ishii, Y. Usuki, and H. Yahagi, Nucl. Instrum. Methods Phys. Res., Sect. A 333, 429 (1993).

3. M. Nikl, Phys. Status Solidi A 178, 595 (2000).

4. A. A. Annenkov, M. V. Korzhik, and P. Lecoq, Nucl. Instrum. Methods Phys. Res., Sect. A 490, 30 (2002).

5. R. W. Novotny, D. Bremer, V. Dormenev, P. Drexler, T. Eissner, T. Kuske, and M. Moritz, J. Phys.: Conf. Ser. 293 (1), 012003 (2011).

6. S. Burachas, M. Ippolitov, V. Manko, S. Nikulin, A. Vasiliev, A. Apanasenko, A. Vasiliev, A. Uzunian, and G. Tamulaitis, Radiat. Meas. 45, 83 (2010).

7. M. Nikl, P. Bohacek, A. Vedda, M. Martini, G. P. Pazzi, P. Fabeni, and M. Kobayashi, Phys. Status Solidi A 182, R3 (2000).

8. A. A. Annenkov, A. E. Borisevich, A. Hofstaetter, M. V. Korzhik, P. Lecoq, V. D. Ligun, O. V. Misevitch, R. Novotny, and J. P. Peigneux, Nucl. Instrum. Methods Phys. Res., Sect. A 450, 71 (2000).

9. M. Kobayashi, Y. Usuki, M. Ishii, M. Itoh, and M. Nikl, Nucl. Instrum. Methods Phys. Res., Sect. A 540, 381 (2005).

10. G.-H. Ren, X.-F. Chen, R.-H. Mao, and D.-Z. Shen, Chin. Phys. (Beijing, China) 59, 4812 (2010).

11. Y. Huang, K. H. Jang, E. S. Kim, L. Shi, and H. J. Seo, J. Korean Phys. Soc. 52, 449 (2008).

12. C. Ye, J. Liao, P. Shao, and J. Xie, Nucl. Instrum. Methods Phys. Res., Sect. A 566, 757 (2006).

13. M. Nikl, P. Bohacek, E. Mihokova, N. Solovieva, A. Vedda, M. Martini, G. P. Pazzi, P. Fabeni, and M. Kobayashi, J. Appl. Phys. 91, 2791 (2002).

14. M. Nikl, P. Bohacek, E. Mihokova, N. Solovieva, A. Vedda, M. Martini, G. P. Pazzi, P. Fabeni, and M. Ishii, J. Appl. Phys. 91, 5041 (2002).

15. R. Wigmans, New J. Phys. 10, 025003 (2008).

16. N. Akchurin, F. Bedeschi, A. Cardini, R. Carosi, G. Ciapetti, M. Fasoli, R. Ferrari, S. Franchino, M. Fraternali, G. Gaudio, J. Hauptman, M. Incagli, F. Lacava, L. La Rotonda, S. Lee, M. Livan, E. Meoni, M. Nikl, D. Pinci, A. Policicchio, S. Popescu, F. Scuri, A. Sill, G. Susinno, W. Vandelli, A. Vedda, T. Venturelli, C. Voena, I. Volobouev, and R. Wigmans, Nucl. Instrum. Methods Phys. Res., Sect. A 621, 212 (2010).

17. E. Auffray, D. Abler, P. Lecoq, and G. Mavromanolakis, IEEE Trans. Nucl. Sci. 57, 1454 (2010).

18. L. L. Nagornaya, F. A. Danevich, A. M. Dubovik, B. V. Grinyov, S. Henry, V. Kapustyanyk, H. Kraus, D. V. Poda, V. M. Kudovbenko, V. B. Mikhailik, M. Panasyuk, O. G. Polischuk, V. Rudyk, V. Tsybulskyi, I. A. Tupitsyna, and Yu. Ya. Vostretsov, IEEE Trans. Nucl. Sci. 56, 2513 (2009).

19. F. A. Danevich, B. V. Grinyov, S. Henry, M. B. Kosmyna, H. Kraus, N. Krutyak, V. M. Kudovbenko, V. B. Mikhailik, L. L. Nagornaya, B. P. Nazarenko, A. S. Nikolaiko, O. G. Polischuk, V. M. Puzikov, A. N. Shekhovtsov, V. I. Tretyak, and Yu. Ya. Vostretsov, Nucl. Instrum. Methods Phys. Res., Sect. A 622, 608 (2010).

20. M. J. J. Lammers, G. Blasse, and D. S. Robertson, Phys. Status Solidi A 63, 569 (1981). 
21. V. Murk, M. Nikl, E. Mihokova, and K. Nitsch, J. Phys.: Condens. Matter 9, 249 (1997).

22. V. V. Laguta, J. Rosa, M. I. Zaritskii, M. Nikl, and Y. Usuki, J. Phys.: Condens. Matter 10, 7293 (1998).

23. V. V. Laguta, M. Martini, F. Meinardi, A. Vedda, A. Hofstaetter, B. K. Mayer, M. Nikl, E. Mihokova, J. Rosa, and Y. Usuki, Phys. Rev. B: Condens. Matter 62, 10109 (2000).

24. V. V. Laguta, M. Martini, A. Vedda, M. Nikl, E. Mihokova, P. Bohacek, J. Rosa, A. Hofstätter, B. K. Meyer, and Y. Usuki, Phys. Rev. B: Condens. Matter 64, 165102 (2001).

25. V. V. Laguta, M. Martini, A. Vedda, E. Rosetta, M. Nikl, E. Mihokova, J. Rosa, and Y. Usuki, Phys. Rev. B: Condens. Matter 67, 205102 (2003).

26. V. V. Laguta, M. Buryi, M. Nikl, J. Rosa, and S. Zazubovich, Phys. Rev. B: Condens. Matter 83, 094123 (2011).

27. S. A. Al'tshuler and B. M. Kozyrev, Electron Paramagnetic Resonance (Academic, London, 1964; Nauka, Moscow, 1972), p. 121.

28. J. Rosa, H. R. Asatryan, and M. Nikl, Phys. Status Solidi B 158, 573 (1996).

29. V. Nistor, M. Stefan, E. Goovaerts, M. Nikl, and P. Bohacek, J. Phys.: Condens. Matter 18, 719 (2006).

30. T. H. Yeom and A. R. Lim, J. Korean Phys. Soc. 49, S562 (2006).
31. S. V. Nistor, M. Stefan, E. Goovaerts, M. Nikl, and P. Bohacek, Radiat. Meas. 38, 655 (2004).

32. M. Stefan, S. V. Nistor, E. Goovaerts, M. Nikl, and P. Bohacek, J. Phys.: Condens. Matter 17, 719 (2005).

33. M. I. Kay, B. C. Frazer, and I. J. Almodovar, J. Chem. Phys. 40, 504 (1964).

34. R. Chipaux, G. Andre, and A. Cousson, J. Alloys Compd. 325, 91 (2001).

35. V. A. Vazhenin, A. P. Potapov, V. B. Guseva, and M. Yu. Artyomov, Phys. Solid State 51 (5), 917 (2009).

36. V. A. Vazhenin, A. P. Potapov, V. B. Guseva, and M. Yu. Artyomov, Phys. Solid State 52 (3), 515 (2010).

37. R. W. Kedzie, D. H. Lyons, and M. Kestigian, Phys. Rev. A 138, 918 (1965).

38. R. M. Golding, M. Kestigian, and C. W. Tennant, J. Phys. C: Solid State Phys. 11, 5041 (1978).

39. D. G. MacGavin and C. W. Tennant, J. Magn. Reson. 61, 321 (1985).

40. R. F. C. Claridge, C. W. Tennant, and D. G. MacGavin, J. Phys. Chem. Solids 58, 813 (1997).

41. Ya. G. Klyava, Electron Paramagnetic Resonance Spectroscopy of Disordered Solids (Zinatne, Riga, 1988), p. 217 [in Russian].

42. T. H. Yeom, J. Korean Phys. Soc. 44, 376 (2004).

Translated by O. Borovik-Romanova 